\title{
Rare Case of Pleomorphic Sarcoma of Oropharynx
}

\author{
Sajal Goel ${ }^{1}$ Deepak K. Mittal ${ }^{1}$ Pankaj Sharma ${ }^{2}$ Nita Khurana ${ }^{3}$ \\ ${ }^{1}$ Department of Clinical Oncology, Delhi State Cancer Institute, \\ Delhi, India \\ 2Department of Radiology, Delhi State Cancer Institute, Delhi, India \\ ${ }^{3}$ Department of Pathology, Maulana Azad Medical College, Delhi, \\ India
}

\begin{abstract}
Address for correspondence Sajal Goel, DNB, Department of Clinical Oncology, Delhi State Cancer Institute (GNCT of Delhi), Dilshad Garden, Delhi 110095, India (e-mail: drsajal.goel@gmail.com).
\end{abstract}

Asian J Oncol 2019;5:72-74

\author{
Abstract \\ Keywords \\ - chronic smoker \\ - pleomorphic sarcoma \\ - oropharynx \\ - nonsurgically
}

Introduction Pleomorphic sarcoma is the commonest soft tissue sarcoma of adult life. Less than $10 \%$ cases of this disease occur as primary in head and neck region. Although a case of pleomorphic sarcoma of lower extremity with metastasis to base of tongue had been reported earlier, a pleomorphic sarcoma arising from oropharynx is being reported for the first time.

Case Report A 75-year-old male chronic smoker was evaluated for complaints of dysphagia, change in voice, and multiple episodes of oral bleeding. He was found to have a locally advanced pleomorphic sarcoma of oropharynx. He was treated nonsurgically. He showed complete clinical and radiologic response. The disease-free survival is 12 months and overall survival is 74 months.

Conclusion This report highlights the importance of nonsurgical management of a case that would have otherwise needed surgery.

\section{Introduction}

Head and neck sarcomas account for approximately $1 \%$ of head/neck malignancies and 5\% of all sarcomas. ${ }^{1,2}$ Pleomorphic sarcoma is the alternate name for malignant fibrous histiocytoma (MFH) as advocated by the World Health Organization (WHO). ${ }^{3,4} \mathrm{MFH}$ is one of the most common adult soft tissue sarcoma. ${ }^{5}$ It can also arise in bones. ${ }^{5}$ It may affect the lower and upper extremities, retroperitoneum, abdominal cavity, and the head and neck, where it accounts for 1 to $3 \%$ of all cases. ${ }^{6}$ In head and neck, it affects in order of frequency: nasal sinus, salivary gland, oral cavity, mandible, larynx, auricula, eyelid, accessory nasal sinuses, and salivary glands. ${ }^{7}$ To the best of our knowledge, oropharynx as primary site is being reported for the first time.

\section{Case History}

A 75-year-old male smoker was evaluated for complaints of dysphagia and change in voice in 3 months' duration with multiple episodes of oral bleeding. Local examination showed a proliferative growth involving posterior tongue, base of tongue, and posterior pharyngeal wall. There were no palpable neck nodes. Contrast-enhanced computed tomography (CECT) of face and neck ( - Fig. 1A, B) showed a bulky necrotic infiltrative heterogeneously enhancing mass of $5 \mathrm{~cm}$ $\times 4.7 \mathrm{~cm} \times 2 \mathrm{~cm}$ size involving base of tongue, extending up to epiglottic tip, and bilateral vallecula, thereby causing significant luminal compromise. Few centimeter-sized bilateral upper cervical lymph nodes were seen. Biopsy (hematoxylin and eosin; - Fig. 2) from primary site proved it pleomorphic sarcoma. Immunohistochemistry showed positivity for vimentin and negativity for epithelial membrane antigen (EMA) and cytokeratin (CK). Metastatic workup with chest $\mathrm{X}$-ray (posteroanterior [PA] view), ultrasound whole abdomen, and CECT chest and abdomen was unremarkable.

He was treated nonsurgically. He received six cycles of neoadjuvant chemotherapy with Injection Ifosfamide $1 \mathrm{~g}$, Carboplatin $100 \mathrm{mg}$, Etoposide $100 \mathrm{mg}$ (Days 1, 8, 15), and Epirubicin $60 \mathrm{mg}$ (Day 1) intravenous (IV) every 22 days. He developed grade-II gastrointestinal and grade-I hematologic toxicities that were managed conservatively. There was complete radiological response on postchemotherapy CECT scan but oral examination showed fullness in bilateral vallecula. Whole body positron emission tomography-computed tomography (WBPET-CT) scan ruled out metastases and demonstrated an ill-defined fluorodeoxyglucose (FDG) avid soft tissue in base of tongue extending to left lateral margin. $\mathrm{He}$ was further taken up for concurrent chemoradiation. He received

June 10, 2019

accepted

October 14, 2019
DOI https://doi.org/

$10.1055 / \mathrm{s}-0039-3400708$ ISSN 2454-6798.
C2019 Spring Hope Cancer Foundation \& Young Oncologist Group of Asia
License terms

(ㅇ) (1) $\ominus \circledast$ 

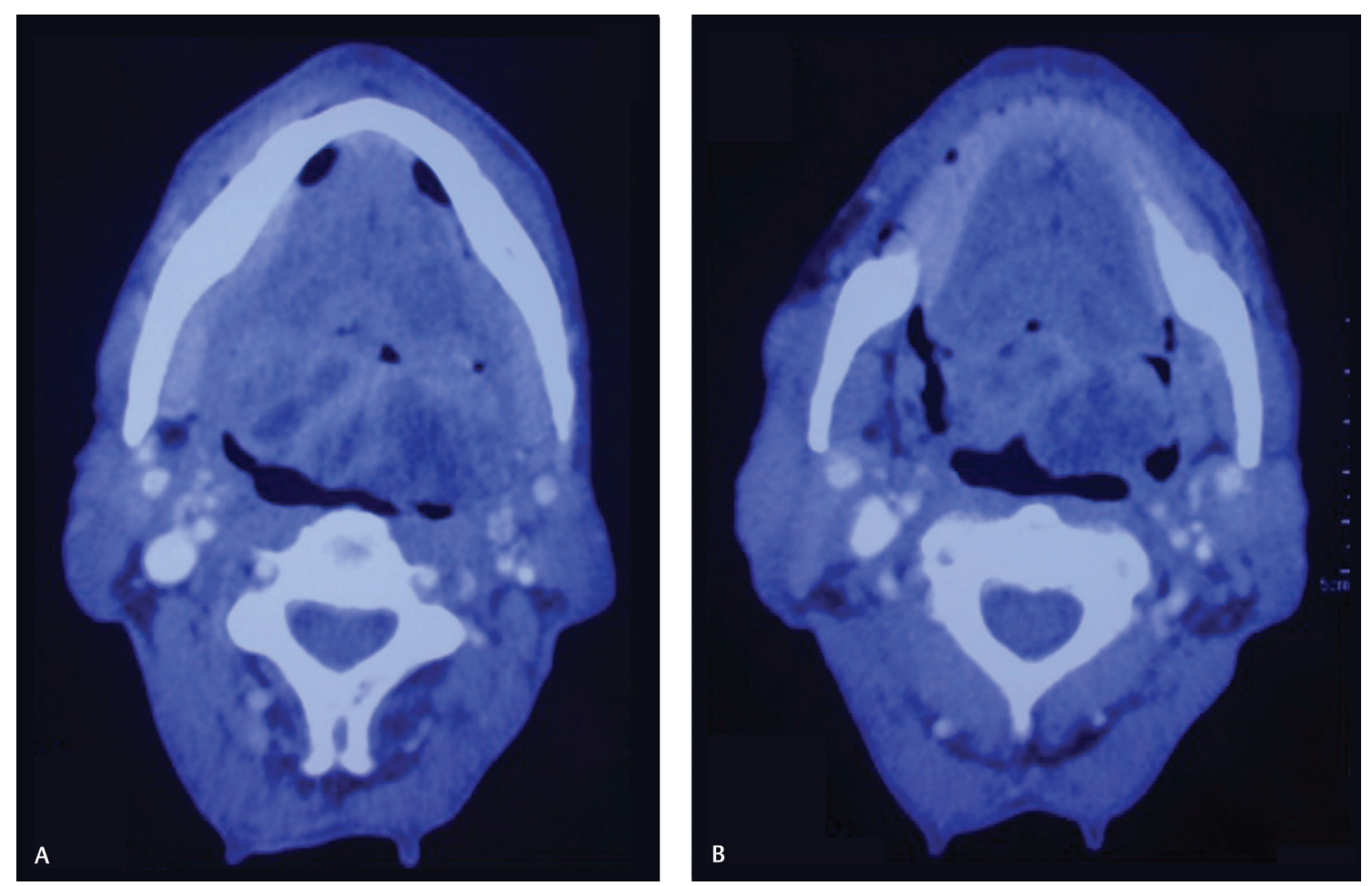

Fig. 1 (A) CECT face and neck (axial view 1), (B) CECT face and neck (axial view 2).

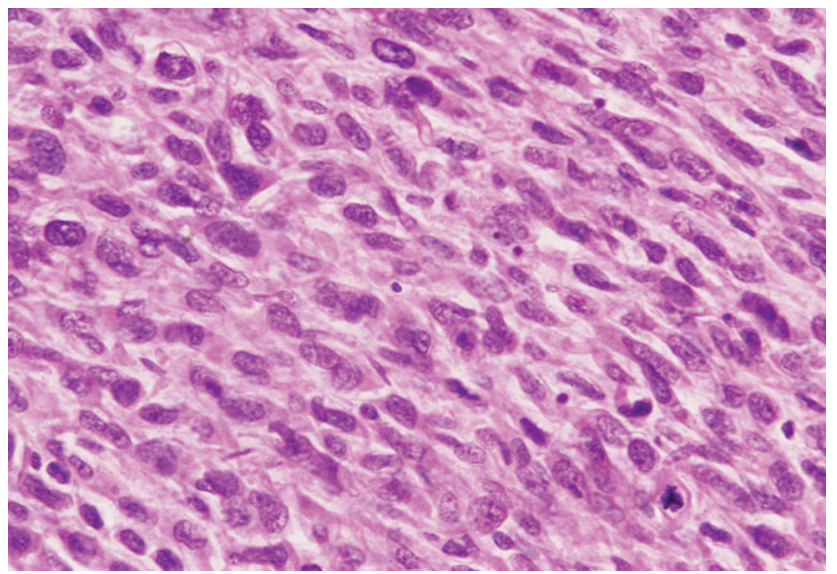

Fig. 2 Sheets of spindle cells showing moderate pleomorphism and high mitosis (hematoxylin and eosin: $\times 600$ ).

received external beam radiotherapy to face and neck by 6 MV photons to a dose of $70 \mathrm{~Gy} / 35$ fractions in 11 weeks by conventional lateral parallel opposed and low-anterior neck portals with shrinking field technique along with concurrent weekly IV cisplatin $50 \mathrm{mg}$ for two cycles. The treatment got prolonged and limited cycles of chemotherapy were given in view of poor tolerance due to grade-III mucositis and dermatitis.

Postradiation CECT scan at 1 month showed a $1.9 \mathrm{~cm} \times 1.2 \mathrm{~cm}$ $\times 1.3 \mathrm{~cm}$ sized residual mass at the base of tongue. He further received two more cycles of same chemotherapy (ICE-Epi). He developed complete clinical response to adjuvant treatment. The patient remained on follow-up. Follow-up WBPET-CT scan at 1 year was normal. Since then, patient is being followed-up regularly. He is locoregionally normal but had xerostomia. The disease-free survival is about 12 months and overall survival is 74 months.

\section{Discussion}

MFH typically occurs in middle-aged adults. The incidence in males is more as compared with female population. ${ }^{5}$ The cause of MFH is unknown, but factors playing role include genetic abnormality on 19p or loss of chromosome 13, radiation treatment for breast cancer, retinoblastoma, Hodgkin's disease, nasopharyngeal carcinomas, and other head and neck malignancies. Signs and symptoms are specific to the location of primary site in head and neck. MFH involving bony structures is more aggressive. ${ }^{8} \mathrm{CT} /$ magnetic resonance imaging (MRI) are diagnostic modalities, although features are nonspecific. ${ }^{5} \mathrm{On}$ CT scan, MFH is usually seen as a large lobulated soft-tissue mass which is isoattenuated to muscle. On MRI, MFH has a heterogeneous hyperintense pattern on T2-weighted images, with an isointensity that is almost the same as that of the muscles on T1-weighted images. ${ }^{9}$

Surgery is the most reliable treatment and radical tumor resection with adequate tumor-free margins is essential. But because of anatomic constraints, surgery is often difficult to perform in head and neck region. ${ }^{5}$ The reported patient was treated nonsurgically with neoadjuvant chemotherapy followed by concurrent chemoradiation.

MFH primarily metastasizes to lung and lymph nodes. The reported frequency of nodal metastases for head and neck MFH varies between 0 and $15 \% .^{5}$ Five-year survival is low in head and neck MFH. ${ }^{5}$ 
The reported case is unique and reveals some interesting facts. Pleomorphic Sarcoma can arise from an unusual location viz. oropharynx in this case. Patient may present as an elderly male and can be cured nonsurgically. A good survival can be achieved with diligent treatment approach and regular follow-up. This case also opens up debate whether the postradiation scan could have been deferred till 12 weeks and better assessment of radiation response could be achieved. Can more patients be treated with nonsurgical approach? Further research on this topic may provide insightful answers.

\section{Conclusion}

This case highlights the importance of unusual primary site, older age of presentation, nonsurgical management, and good overall survival of a case of oropharyngeal pleomorphic sarcoma. This case also highlights that concurrent chemoradiation can be given in such a case if surgical option is unavailable and patient should not be left deprived of treatment.

\section{Note}

This case was presented at ISMPOCON (poster presentation), Delhi, on November 6, 2016.

\section{Conflict of Interest}

None declared.

\section{Acknowledgment}

The authors acknowledge the efforts of Dr. Nita Khurana for pathological diagnosis.

\section{References}

1 Potter BO, Sturgis EM. Sarcomas of the head and neck. Surg Oncol Clin N Am 2003;12(2):379-417

2 Hoffman HT, Robinson RA, Spiess JL, Buatti J. Update in management of head and neck sarcoma. Curr Opin Oncol 2004;16(4):333-341

3 Al-Agha OM, Igbokwe AA. Malignant fibrous histiocytoma: between the past and the present. Arch Pathol Lab Med 2008;132(6):1030-1035

4 Fletcher CDM, Unni KK, Mertens F. Pathology and Genetics of Tumours of Soft Tissue and Bone. IARC Press: Lyon; 2002: $120-125$

5 Gonzalez-Gonzalez R, Bologna-Molina R, Tremillo-Maldonado O, Carreon-Burciaga RG, Palacio-Gastelúm MG. Head and neck soft tissue sarcoma. In: Olivers CL, ed. Soft Tissue Tumors. London: InTech Open; 2011:117-143

6 Gibbs JF, Huang PP, Lee RJ, et al. Malignant fibrous histiocytoma: an institutional review. Cancer Invest 2001;19(1):23-27

7 Barnes L, Kanbour A. Malignant fibrous histiocytoma of the head and neck. A report of 12 cases. Arch Otolaryngol Head Neck Surg 1988;114(10):1149-1156

8 Rinaldo A, Shaha AR, Pellitteri PK, Bradley PJ, Ferlito A. Management of malignant sublingual salivary gland tumors. Oral Oncol 2004;40(1):2-5

9 Park SW, Kim HJ, Lee JH, Ko YH. Malignant fibrous histiocytoma of the head and neck: CT and MR imaging findings. Am J Neuroradiol 2009;30(1):71-76 